\title{
ENGORDA DE CORDEROS PELIBUEY CON DIFERENTE NIVEL DE ALFALFA EN LA DIETA
}

\author{
FATTENING PELIBUEY LAMBS FED WITH DIFFERENT ALFALFA LEVELS IN THE DIET
}

Resendiz, C.V. ${ }^{1}$; Hernández, O.. ${ }^{1 *}$ Guerrero, I.2; Gallegos, J. ${ }^{1}$ Martínez, P.A. ${ }^{3}$ y Sánchez, C. ${ }^{3}$

'Programa de Ganadería-Colegio de Postgraduados. Montecillo, Estado de México. México. *ohmendo@colpos.mx

${ }^{2}$ Departamento de Biotecnología. Universidad Autónoma Metropolitana-Iztapalapa. Iztapalapa. México, D.F. México.

${ }^{3}$ Departamento de Zootecnia. Universidad Autónoma Chapingo. Chapingo. México.

\section{Palabras clave adicionales}

Ganancia de peso vivo. Rendimiento de canal.

\section{RESUMEN}

El objetivo de este estudio fue evaluar el efecto de diferentes niveles de alfalfa (Medicago sativa L.) en la dieta de corderos sobre el comportamiento productivo, durante 11 semanas. Se utilizaron 36 corderos machos Pelibuey de 5 meses de edad, en estabulación, con peso inicial promedio de 22 $\mathrm{kg}$, distribuidos homogéneamente en cuatro grupos de 3 animales cada uno, con 3 repeticiones por grupo. Los grupos fueron asignados al azar a cada uno de cuatro tratamientos evaluados: 0 , 20,30 y $40 \%$ de alfalfa (base seca). Se utilizó un diseño completamente al azar, utilizando el Proc GLM (SAS, 1999) y cuando se observaron diferencias estadísticas, se realizó una comparación de medias utilizando la prueba de Tukey. Se determinó consumo de alimento, ganancia de peso, conversión, eficiencia alimenticia, rendimiento de canal en caliente y frio, y pH de la canal; además de la degradabilidad in situ de la MS de las dietas. El consumo de MS aumentó $(p<0,05)$ de 1,19 a 1,39 $\mathrm{kg} \mathrm{MS}$ animal ${ }^{-1}$ día $^{-1}$ al incrementar el nivel de alfalfa de 20 a $40 \%$, respectivamente, a pesar que las dietas que contenían alfalfa tuvieron menor $(p<0,05)$ degradabilidad en comparación con la dieta testigo; sin embargo, la ganancia diaria de peso no se afectó $(p>0,05)$, promediando $271 \mathrm{~g}$ animal ${ }^{-1}$ día $^{-1}$. Tampoco hubo variación $(p>0,05)$ en el peso final, conversión y eficiencia alimenticia, promediando $38,0 \mathrm{~kg}^{2}$ animal-1 $^{-1} 4,74$ y 0,212 , respectivamente. El peso $(19,3$ y $18,8 \mathrm{~kg})$ y rendimiento $(54,4$ y $53,0 \%)$ de canal en caliente y fría, fueron

\author{
AdDitionAL KEYWORDS \\ Carcass yield. Live-weight gain.
}

similares entre tratamientos. Incluir alfalfa en la dieta de corderos, no ofrece ventajas en la ganancia de peso, sin embargo hasta $40 \%$, podría ser atractivo económicamente para el productor, además de promover mayor consumo de MS.

\section{SUMMARY}

The objective of this study was to evaluate the effect of feeding alfalfa (Medicago sativa L.) to lambs on animal performance, during a period of 11 weeks. Thirty-six, 5 months old, Pelibuey male lambs were used indoors, with initial live weight of $22.0 \mathrm{~kg}$. They were distributed homogeneously into four groups of three each, with three replicates per group, and then randomly assigned to each of the following treatments: diet with $0,20,30$ and 40 $\%$ (dry basis) of alfalfa. Dry matter intake (DMI), daily gain weight (DGW), feeding conversion (FC) and efficiency (FE), hot and cold carcass yield, biological hot and cold carcass yield, and carcass $\mathrm{pH}$ at slauthering and $24 \mathrm{~h}$ post mortem, and digestibility in situ of the diet, were evaluated. A completely randomized design using Proc GLM was used; mean comparison was done using the Tukey test. There were not significant differences $(p>0.05)$ between treatments on animal performance, except on dry matter intake, which increased $(p<0.05)$ from 1.2 to $1.4 \mathrm{~kg}$ DM per animal when increasing alfalfa level from 20 to $40 \%$, even though diets including alfalfa had less 


\section{RESENDIZ, HERNÁNDEZ, GUERRERO, GALLEGOS, MARTÍNEZY SÁNCHEZ}

digestibility compared to the control one. DGW, FC and FE, averaged $271 \mathrm{~g}^{\text {animal }}{ }^{-1}$ day $^{-1}, 4.74$ and 0.212 , respectively. The average for hot and cold carcass yield, were $54.4 \%$ and $53.0 \%$, respectively. Including alfalfa in the lamb diet, offers no benefits on animal performance; however, including up to $40 \%$ of alfalfa, could be a viable feeding strategy, and from the economical point of view could be attractive to the farmer, besides, it increases dry matter intake.

\section{INTRODUCCIÓN}

La producción ovina en México tiene como objetivo principal la obtención de carne (López et al., 2000). Recientemente se ha propiciado mayor desarrollo de la ovinocultura en diferentes regiones del país, donde las razas de pelo, como la Pelibuey, han cobrado importancia dadas sus características favorables de rusticidad, manejo y productividad (Morales et al., 2004; Medrano, 2000). Sin embargo, la demanda nacional de carne ovina no se ha logrado cubrir, por lo que ha sido necesaria la importación, que en 2005 fue alrededor de 46,2\% del consumo nacional aparente (SAGARPA, 2010). Además, la explotación ovina, como las otras, enfrenta el problema del alto costo de producción por concepto de alimentación, especialmente en los sistemas intensivos, donde los granos son la base de la alimentación, principalmente maíz, cuyo elevado precio agudiza el problema (Pérez et $a l ., 2010$ ), por lo que los forrajes se presentan como una alternativa viable en la alimentación de rumiantes (Martin y Rogers, 2004). La alfalfa ocupa un espacio preponderante, por ser una especie forrajera con alto potencial productivo que, en la última década a nivel nacional, aumentó en 27,2 \% (SIAP, 2010), lo que es especialmente importante porque la alfalfa constituye un recurso forrajero de 18,4 a $20 \%$ de proteína (Bhatti et al., 2008; Jahn et al., 2000). Su uso es amplio en ganado lechero como forraje de excelente calidad, utilizándose en monocultivo o en asociación, en pastoreo o bien como forraje de corte fresco o henificado
(Brito et al., 2009; Getachew et al., 2006), habiendo más estudios orientados a producción y composición de la leche (Álvarez et al., 2006; Moss et al., 2002). En ovinos, la alfalfa se relaciona más con estudios enfocados a aspectos agronómicos (Mendiola et al., 2007; Tablada et al., 2003), pero pocos a la respuesta en comportamiento productivo en la etapa de engorda de los animales. Recientemente, estudios realizados en México muestran ganancias de peso y rendimiento en canal caliente en borregos Pelibuey, de 90 a $181 \mathrm{~g}_{\text {animal }}{ }^{-1}$ día ${ }^{-1}$, y de 50,79 a $54,90 \%$, respectivamente (Partida et al., 2009; Jaramillo et al., 2008; Álvarez et al., 2003), con diferentes regímenes alimenticios en condiciones de estabulación. Estos resultados podrían mejorarse con una estrategia de alimentación adecuada, donde se considere la máxima inclusión de forrajes en la dieta para disminuir los costos de producción, y obtener un producto de calidad como valor agregado del sistema.

El objetivo de este trabajo fue determinar la magnitud de los indicadores biológicos y económicos de la engorda de corderos Pelibuey alimentados con dietas con diferente nivel de alfalfa.

\section{MATERIAL Y MÉTODOS}

El estudio se realizó de agosto a noviembre de 2009, en el Módulo de Producción Ovina de la Granja Experimental de la Universidad Autónoma Chapingo, en Chapingo, Estado de México, México, ubicado a $19^{\circ} 29^{\prime}$ latitud norte, $98^{\circ} 54^{\prime}$ longitud oeste, a 2240 msnm. El clima es templado subhúmedo con lluvias en verano, la precipitación promedio anual es de $644,8 \mathrm{~mm}$, y la temperatura media anual de $15^{\circ} \mathrm{C}$ (García, 1989).

Se utilizaron 36 corderos Pelibuey, de 5 meses y peso promedio inicial de $22 \mathrm{~kg}$, distribuidos homogéneamente de acuerdo a su peso vivo en 4 grupos de 3 animales cada uno, con 3 repeticiones, considerando cada grupo como una repetición. Los grupos de animales se asignaron al azar a cada 


\section{USO DE LA ALFALFA EN LA ALIMENTACIÓN DE CORDEROS PELIBUEY}

uno de los 4 tratamientos evaluados, que consistieron en dietas con $0,20,30$ y $40 \%$ de alfalfa sobre base seca (tabla I). La dietas se formularon de acuerdo con los requerimientos nutricionales para ovinos (NRC, 2007), usando el software UFFDA (User Friendly-Feed Formulation). El alimento se ofreció tres veces al día, 7:00 am, 1:00 y 7:00 pm, en una proporción de 60, 20 y $20 \%$ respectivamente, con agua ad libitum, con un periodo de adaptación de 15 días.

Las variables medidas fueron: peso inicial, peso final, consumo de materia seca, ganancia diaria de peso, conversión y eficiencia alimenticia; así como también, peso de cabeza, sangre, piel, extremidades, vísceras, peso vivo vacío, peso de la canal, rendimiento de canal, rendimiento biológico y $\mathrm{pH}$.

El peso inicial y final se obtuvieron al entrar y salir de la fase experimental. El consumo de alimento fue la diferencia de la cantidad de alimento ofrecido y el rechazado diariamente. La ganancia diaria de peso se calculó con base al promedio de la ganancia diaria de peso de 6 pesajes realizados durante la fase experimental, realizadas cada 15 días. La conversión alimenticia se calculó dividiendo el consumo de materia seca entre la ganancia diaria de peso, y la eficiencia alimenticia, dividiendo la ganancia diaria de peso entre el consumo diario de materia seca.

Además, se determinó la degradabilidad y análisis químico de la materia seca de cada una de las dietas. La degradabilidad se realizó in situ, utilizando 4 borregos con cánula ruminal, alimentados con heno de alfalfa y agua ad libitum. Para ello, se utilizó un diseño cuadrado latino 4 x 4. Se emplearon bolsas $(3 \times 5 \mathrm{~cm})$ de forro japonés con poro de $52 \mathrm{~mm}$, las muestras de alimento previamente fueron molidas con una malla de 2 $\mathrm{mm}$. Se colocaron $3 \mathrm{~g}$ de muestra de cada dieta en cada bolsa, con 3 repeticiones por cada tiempo de incubación, sujetándolas a una cadena de acero inoxidable. Las bolsas fueron incubadas a $0,6,12,24,48$ y $72 \mathrm{~h}$ en
Tabla I. Ingredientes y composición química de las dietas consumidas por corderos Pelibuey. (Ingredients and chemical composition of the experimental diets used in Pelibuey lambs feeding).

\begin{tabular}{lcccc}
\hline & \multicolumn{4}{c}{ Nivel de alfalfa (\%) } \\
& 0 & 20 & 30 & 40 \\
\hline Ingredientes (\%) & & & & \\
Alfalfa & 0 & 19,98 & 29,97 & 40,02 \\
Sorgo & 50,29 & 43,50 & 38,58 & 32,93 \\
Pasta de soya & 14,04 & 9,06 & 6,33 & 4,49 \\
Rastrojo de maíz & 11,99 & 6,00 & 2,97 & 0,00 \\
Cascarilla de soya & 7,99 & 6,00 & 6,03 & 5,99 \\
Salvado de trigo & 7,50 & 6,00 & 6,03 & 5,99 \\
Melaza & 4,97 & 5,02 & 5,04 & 4,99 \\
Minerales* & 1,46 & 1,48 & 1,48 & 1,50 \\
Carbonato de calcio & 0,97 & 0,98 & 0,99 & 1,00 \\
Urea & 0,49 & 0,49 & 0,49 & 0,40 \\
Aceite crudo de soya & 0,29 & 1,48 & 2,08 & 2,69 \\
& & & & \\
Composición química & & & & \\
Materia seca (\%) & 87,2 & 86,8 & 86,9 & 86,3 \\
Proteína (\%) & 15,6 & 16,0 & 16,4 & 15,9 \\
FDN (\%) & 40,37 & 39,47 & 35,57 & 37,11 \\
FDA (\%) & 12,60 & 15,50 & 17,77 & 18,88 \\
Cenizas (\%) & 5,6 & 6,6 & 7,9 & 8,4 \\
\hline
\end{tabular}

*Vitasal ovino plus: calcio, $24 \%$; fosforo, $3 \%$; magnesio, $2 \%$; sodio, $8 \%$; cloro, $12 \%$; potasio, $0,5 \%$; azufre, $0,5 \%$; antioxidante, $0,5 \%$; lasolacida, 2000 ppm; cromo, 5 ppm; manganeso, 4000 ppm; hierro, 2000 ppm; cinc, 5000 ppm; yodo, 100 ppm; selenio, 30 ppm; cobalto, 60 ppm; vit. A, 500000 Ul; vit. D, 150000 Ul; vit. E, 1000 UI.

FDN= fibra detergente neutro; FDA = fibra detergente ácido.

el rumen, siendo colocadas en sentido inverso al citado para ser retiradas todas al mismo tiempo. Una vez retiradas, las muestras se lavaron con agua corriente hasta que ésta fue clara. Las muestras se secaron a temperatura ambiente, y posteriormente en una estufa de aire forzado a $55^{\circ} \mathrm{C}$ por 24 horas. La degradabilidad de la materia seca se determinó como el porcentaje de muestra desaparecida en la bolsa.

El análisis químico se realizó en el Labo- 


\section{RESENDIZ, HERNÁNDEZ, GUERRERO, GALLEGOS, MARTíNEZ Y SÁNCHEZ}

ratorio de Nutrición Animal del Colegio de Postgraduados. Se tomaron muestras de alimento por tratamiento cada 15 días, mezclándose al finalizar el experimento, para obtener una sola muestra por tratamiento, a las cuales se les determinó materia seca, proteína cruda (AOAC, 1984), fibra detergente neutro y ácido (Van Soest et al., 1991), cenizas (AOAC, 1984) y degradabilidad in situ.

Al completar 11 semanas del periodo de engorda, los animales fueron enviados al matadero con un peso vivo promedio de 38 $\mathrm{kg}$. Después de un periodo de ayuno de 24 $\mathrm{h}$, los borregos fueron pesados para obtener el peso vivo al sacrificio (PVS). Posteriormente, los animales se sacrificaron, retirando y pesando la sangre, cabeza, extremidades (patas y manos), piel y vísceras, y canal caliente (PCC). Paralelamente se pesaron las vísceras llenas y después se vaciaron, y por diferencia se estimó el peso del contenido digestivo constituyendo el peso vivo vacío (PVV). Posteriormente las canales se guardaron en una cámara frigorífica a una temperatura de $4{ }^{\circ} \mathrm{C}$. Transcurridas 24 horas del sacrificio, la canal fría se pesó (PCF) y se tomó una muestra del solomillo, la cual fue etiquetada, empaquetada al vacío y almacenada en congelación para su posterior análisis.

El pH y la temperatura de la canal se registraron al momento del sacrificio y a las 24 horas post mórtem, utilizando un potenciómetro portátil (HANNA, mod. HI99163) con un electrodo de penetración, la medición fue entre la $12^{\mathrm{a}}$ y $13^{\mathrm{a}}$ costilla, directamente en la canal.

Con los datos obtenidos durante el sacrificio se registró el rendimiento de la canal caliente [(PCC/PVS $) \times 100]$, rendimiento de la canal fría [(PCF/PVS $) \times 100]$, rendimiento biológico de la canal caliente [(PCC/PVV) $\mathrm{x}$ 100] y el rendimiento biológico de la canal fría [(PCF/PVV) x 100] (Hernández et al., 2009).

Los análisis estadísticos se realizaron utilizando el procedimiento GLM del paque- te estadístico SAS (1999). Para la comparación de medias de tratamientos se utilizó la prueba de Tukey (Steel y Torrie, 1989). El nivel de alfalfa constituyó el factor de interés tomando el animal como unidad experimental.

\section{RESULTADOSYDISCUSIÓN}

\section{DEGRADABILIDAD DE LA MATERIA SECA DE LAS DIETAS}

En la tabla II se presentan los resultados de la degradabilidad de las dietas. Se encontraron diferencias estadísticas $(\mathrm{p}<0,05)$ entre tratamientos en todos los tiempos de incubación, a excepción de las 0 horas ( $>>0,05)$. A las 72 horas se observó menor ( $\mathrm{p}<0,0001)$ degradabilidad conforme aumentó el nivel de alfalfa, cuyos promedios fueron 92,$4 ; 90,8 ; 86,9$ y $87,7 \%$, para $0,20,30$ y $40 \%$ de alfalfa, respectivamente. Esta disminución se debió básicamente al incremento de la fibra de la dieta, al aumentar la cantidad de lignina debido a la alfalfa, ya que ésta, junto con la celulosa, forman una barrera que sirve de protección al ataque de microorganismos, reduciendo así la digestibilidad (McDonald et al., 2006; Barahona y Sánchez, 2005). Contrariamente, en la die-

Tabla II. Degradabilidad in situ (\%) de la materia seca de las dietas a diferentes horas de incubación. (Dry matter in situ degradability (\%) of diets at different hours of incubation).

\begin{tabular}{lcccccc}
\hline \multicolumn{7}{c}{ Tratamientos } \\
$\mathrm{h}$ & 0 & 20 & 30 & 40 & $\mathrm{DE}$ & $\mathrm{p}$ \\
\hline 0 & $15,2^{\mathrm{a}}$ & $16,4^{\mathrm{a}}$ & $13,6^{\mathrm{a}}$ & $14,0^{\mathrm{a}}$ & 3,9 & 0,2894 \\
6 & $52,5^{\mathrm{a}}$ & $51,9^{\mathrm{ab}}$ & $44,6^{\mathrm{b}}$ & $46,7^{\mathrm{ab}}$ & 7,7 & 0,0198 \\
12 & $66,6^{\mathrm{a}}$ & $59,5^{\mathrm{ab}}$ & $52,3^{\mathrm{b}}$ & $59,1^{\mathrm{ab}}$ & 8,7 & 0,0003 \\
24 & $84,2^{\mathrm{a}}$ & $75,0^{\mathrm{b}}$ & $74,6^{\mathrm{b}}$ & $74,4^{\mathrm{b}}$ & 8,3 & 0,0046 \\
48 & $90,2^{\mathrm{a}}$ & $87,1^{\mathrm{ab}}$ & $83,6^{\mathrm{b}}$ & $85,2^{\mathrm{ab}}$ & 5,2 & 0,0082 \\
72 & $92,4^{\mathrm{a}}$ & $90,8^{\mathrm{b}}$ & $86,9^{\mathrm{c}}$ & $87,7^{\mathrm{c}}$ & 2,5 & 0,0001
\end{tabular}

$\mathrm{DE}=$ desviación estándar.

${ }^{a b c}$ En cada fila, cifras con letras distintas son significativamente diferentes. 


\section{USO DE LA ALFALFA EN LA ALIMENTACIÓN DE CORDEROS PELIBUEY}

ta testigo, donde no se incluyó alfalfa, la mayor degradabilidad estuvo definida por la cantidad de sorgo, que presenta una digestibilidad de 76,0\% (Mora et al., 2002), mayor a 59,9 \% de la alfalfa (Avendaño et al., 2004). Estos resultados coinciden con los de Phillips et al. (2002), quienes reportan que al aumentar el porcentaje de pellet de alfalfa y disminuir kenaf (Hibiscus cannabinus), el porcentaje de la digestibilidad disminuyó, atribuyéndolo a menor digestibilidad de la FDN y mayor contenido de FDA de la alfalfa, comparado con la del kenaf.

\section{COMPORTAMIENTOPRODUCTIVO}

Los niveles de alfalfa no afectaron ( $>>0,05)$ los parámetros de producción, excepto consumo de alimento $(\mathrm{p}<0,05)$ (tabla III). Los animales que recibieron la dieta con $40 \%$ de alfalfa tuvieron un mayor consumo, seguidos por los que recibieron 30, 0 y $20 \%$, respectivamente. Estos resultados coinciden con los de Fernández et al. (2004), quienes señalan que cuando se incrementó de 0 a $90 \%$ de heno de alfalfa peletizado en la dieta de ovinos, el consumo de alimento aumentó de 1,0 a 1,9 kg animal ${ }^{-1}$ día $^{-1}$, respectivamente. Pareciera contradictorio que los animales que recibieron la dieta con $40 \%$ de alfalfa, que mostró menor degradabilidad, presentaran mayor consumo. Al respecto, es pertinente mencionar que tal degradabilidad es referida a las muestras íntegras de cada dieta, mientras que el consumo aquí presentado es referido a la fracción que los animales efectivamente consumieron, ya que éstos presentaron alto grado de selectividad, que se observó al dejar alrededor de 20$30 \%$ de los tallos de alfalfa en el residual, componente que tiene la mayor parte de la fracción no digestible, y que no es preferida por el animal (Ramírez et al., 2003). Ello indica mayor consumo de hojas, provocando mayor velocidad de paso por el rumen que el tallo (Boschini, 2001), y consecuentemente, una disminución en el tiempo de retención del alimento, propiciando mayor consumo (Whetsell et al., 2004). Además, es posible que el mayor consumo de las dietas que contenían alfalfa, haya sido por una mejor apetecibilidad de la misma (Bhatti et al., 2008; Distel y Villalba, 2007), en comparación con la dieta testigo, ya que ésta última contenía mayor porcentaje de sorgo, cuyo contenido de taninos es de $1,5 \%$ en promedio (Ojeda et al., 2010), confiriendo al alimento un sabor astringente (Church et al., 2007; Martínez et al., 2000), y por tanto su rechazo. Esta situación no sucede cuando se incluye alfalfa, ya que según Otero e Hidalgo (2004), la alfalfa contiene solo 0,05

Tabla III. Parámetros de producción de ovinos Pelibuey alimentados con diferentes niveles de alfalfa en la dieta. (Productive parameters of Pelibuey sheep fed with different levels of alfalfa in the diet).

\begin{tabular}{lcccccc}
\hline & \multicolumn{7}{c}{ Tratamientos $(\%$ de alfalfa $)$} & & \\
Variable & 0 & 20 & 30 & 40 & DE & Sig. \\
\hline Peso vivo inicial $(\mathrm{kg})$ & $22,40^{\mathrm{a}}$ & $22,34^{\mathrm{a}}$ & $22,31^{\mathrm{a}}$ & $22,30^{\mathrm{a}}$ & 0,260 & $\mathrm{NS}$ \\
Peso vivo final $(\mathrm{kg})$ & $37,79^{\mathrm{a}}$ & $37,52^{\mathrm{a}}$ & $37,97^{\mathrm{a}}$ & $38,80^{\mathrm{a}}$ & 0,966 & $\mathrm{NS}$ \\
Consumo de MS $(\mathrm{kg})$ & $1,20^{\mathrm{ab}}$ & $1,19^{\mathrm{b}}$ & $1,36^{\mathrm{ab}}$ & $1,39^{\mathrm{a}}$ & 0,115 & 0,0191 \\
Ganancia diaria de peso $(\mathrm{kg})$ & $0,260^{\mathrm{a}}$ & $0,250^{\mathrm{a}}$ & $0,293^{\mathrm{a}}$ & $0,280^{\mathrm{a}}$ & 0,024 & $\mathrm{NS}$ \\
Conversión alimenticia & $4,61^{\mathrm{a}}$ & $4,76^{\mathrm{a}}$ & $4,62^{\mathrm{a}}$ & $4,98^{\mathrm{a}}$ & 0,273 & $\mathrm{NS}$ \\
Eficiencia alimenticia & $0,217^{\mathrm{a}}$ & $0,213^{\mathrm{a}}$ & $0,217^{\mathrm{a}}$ & $0,200^{\mathrm{a}}$ & 0,013 & $\mathrm{NS}$ \\
\hline
\end{tabular}

$\mathrm{DE}=$ desviación estándar; Sig.= significancia.

${ }^{a b}$ En cada fila, cifras con letras distintas son significativamente diferentes. 


\section{RESENDIZ, HERNÁNDEZ, GUERRERO, GALLEGOS, MARTÍNEZ Y SÁNCHEZ}

$\%$ de taninos, cantidad insuficiente para provocar aversión. Al respecto, Larraín et al. (2009) observaron un menor consumo de alimento en toros alimentados con dietas altas en taninos, repercutiendo en una menor ganancia de peso. Adicionalmente, se ha reportado que los ácidos grasos volátiles (AGV) están involucrados en el proceso de consumo de materia seca, particularmente, el propiónico (Loncke et al., 2009; Krizsan y Randby, 2007) que actúa como sustrato fundamental en la gluconeogénesis, proceso por el cual los rumiantes obtienen glucosa a partir de compuestos que no son carbohidratos (Murray et al., 2007). En este estudio, la concentración de propiónico disminuyó (13,14 mmol/L) cuando se incluyó alfalfa en la dieta, en comparación con la dieta testigo $(18,21 \mathrm{mmol} / \mathrm{L})$, y podría ser ésta la razón de mayor consumo de las dietas con alfalfa, aunque Mbanya et al., 1993 (citado por Krizsan y Randby, 2007) mostraron lo contrario en bovinos productores de leche, cuando el ácido acético y propiónico fueron infundidos por separado.

La ganancia diaria de peso fue similar entre tratamientos, promediando $271 \mathrm{~g}$ ani$\mathrm{mal}^{-1} \mathrm{dí}^{-1}$, superior a la reportada por Partida et al. (2009), Álvarez et al. (2003) y Marshall et al. (2001), quienes encontraron valores promedio de 181,90 y $110 \mathrm{~g}_{\text {animal }}{ }^{-1}$ día ${ }^{-1}$ en borregos Pelibuey, respectivamente. En el presente estudio, a diferencia de los mencionados anteriormente, el alimento se ofreció 3 veces al día, estimulando mayor consumo de materia seca, probablemente por ofrecerlo más fresco, haciéndolo más apetecible, y consecuentemente, mayor producción. Adicionalmente, según Partida et al. (2009), deben tomarse en consideración los efectos raza y sexo, pues ellos, reportaron ganancias diarias de peso de 206 y 222 g animal ${ }^{-1}$ día $^{-1}$ en borregos Pelibuey cruzados con Suffolk y Dorset, respectivamente. El hecho de no haber encontrado diferencias significativas entre tratamientos en comportamiento productivo, sugiere que la alfalfa puede ser una alternativa viable en la engorda de borregos, especialmente porque se obtienen ganancias superiores a las reportadas, incluso a las señaladas por el NRC. Esto adquiere especial importancia si se considera que los animales utilizados fueron de pelo, considerados con menores ganancias de peso, aunque mejor adaptadas a condiciones adversas, donde otras razas no proliferan (López et al., 2000).

La conversión y eficiencia alimenticia fueron similares $(\mathrm{p}>0,05)$ entre tratamientos, con promedios de 4,74 y 0,212 , respectivamente. Estos resultados son menores a los obtenidos por Partida et al. (2009) y Álvarez et al. (2003), quienes encontraron valores de 6,6 y 8,6, respectivamente para conversión alimenticia en borregos Pelibuey, en tanto que Álvarez et al. (2003), reportaron una eficiencia alimenticia de 0,137 . Esto sugiere que utilizar alfalfa como ingrediente en la dieta, pudo influir en una menor conversión, dado que a pesar de que las dietas fueron isoproteicas, la alfalfa tiene la ventaja de mayor contenido de aminoácidos que llegan a duodeno para su absorción, a pesar de que su proteína es altamente degradada en rumen (Getachew et al., 2006). Es importante señalar que las dietas utilizadas por Partida et al. (2009) y Álvarez et al. (2003) contenían 14,0 y 11,6\% de proteína bruta, respectivamente, comparado con $16 \%$ en promedio, en las dietas utilizadas en el presente experimento. Según Getachew et al. (2006) la proteína que escapa a la degradación ruminal, se ve afectada por su naturaleza y por su cantidad en la dieta. Ellos reportan que incrementar el contenido de proteína bruta de 13,1 a $23 \%$, aumenta la proporción de aminoácidos que llegan a duodeno de 49 a $65 \%$. Adicionalmente, Haugen et al. (2006) señalan que la degradación de la proteína de heno de alfalfa, se ve disminuida por la reducción de la solubilidad de las proteínas citoplasmáticas, situación que pudo suceder con la alfalfa, henificada, utilizada en el presente estudio. Asimismo, la degradabilidad de las dietas fue mayor $(89,4 \%)$, que la indicada (85\%) por Álvarez 


\section{USO DE LA ALFALFA EN LA ALIMENTACIÓN DE CORDEROS PELIBUEY}

et al. (2003). Esto podría justificar los resultados aquí reportados, que muestran que al incluir alfalfa hasta en $40 \%$ de la dieta y disminuir el sorgo en $18 \%$ aproximadamente ( 0 vs. $40 \%$ de alfalfa), el CMS, GDP, CA y EA, son similares, lo cual indica que se cubren los requerimientos nutricionales para obtener en promedio $271 \mathrm{~g}_{\text {animal }}{ }^{-1} \mathrm{día}^{-1}$.

La importancia de estos resultados radica en el beneficio que representaría para el productor, al utilizar menor cantidad de alimento para obtener un $\mathrm{kg}$ de peso vivo comparado con otros reportes, y en cierto grado, representaría beneficios económicos por concepto de alimentación.

\section{Rendimientode canal}

Los diferentes niveles de alfalfa inclui- dos en la dieta de corderos, no afectaron $(p>0,05)$ a las características de la canal, ni al porcentaje de cabeza, sangre, piel, vísceras (verdes y rojas) y $\mathrm{pH}$, pero sí $(\mathrm{p}<0,05)$ al porcentaje de extremidades (tabla IV), el cual fue mayor con $30 \%$ de alfalfa, seguido de aquellos con 20, 40 y $0 \%$ de alfalfa, respectivamente.

El peso $(19,3 \mathrm{~kg})$ y el rendimiento $(54,4 \%)$ de canal caliente no fueron diferentes entre tratamientos siendo mayores que los reportados por Jaramillo et al. (2008) y Marshall et al. (2001), pero menores que los de Torrescano et al. (2009) y Partida et al. (2009). Estudios recientes han demostrado que borregos finalizados con concentrados presentan mayor rendimiento en canal que los finalizados en pastoreo (Borton et al.,

Tabla IV. Rendimiento de la canal de borregos Pelibuey alimentados con dietas con diferentes niveles de alfalfa. (Carcass yield of Pelibuey sheep fed with different levels of alfalfa in the diet).

\begin{tabular}{|c|c|c|c|c|c|c|}
\hline \multirow[b]{2}{*}{ Variable } & \multicolumn{4}{|c|}{ Tratamientos (\% de alfalfa) } & \multirow[b]{2}{*}{$\mathrm{DE}$} & \multirow[b]{2}{*}{ Sig. } \\
\hline & 0 & 20 & 30 & 40 & & \\
\hline Peso vivo (kg) & $35,77^{a}$ & $34,91^{\mathrm{a}}$ & $35,30^{a}$ & $35,81^{a}$ & 1,24 & NS \\
\hline PVV (kg) & $31,87^{a}$ & $31,00^{a}$ & $31,56^{a}$ & $31,47^{a}$ & 1,06 & NS \\
\hline $\mathrm{PCC}(\mathrm{kg})$ & $19,72^{a}$ & $18,84^{a}$ & $19,18^{a}$ & $19,37^{a}$ & 0,58 & NS \\
\hline PCF (kg) & $19,06^{a}$ & $18,49^{a}$ & $18,78^{a}$ & $18,73^{a}$ & 0,56 & NS \\
\hline $\mathrm{RCC}(\%)$ & $55,13^{a}$ & $54,00^{a}$ & $54,30^{a}$ & $54,15^{a}$ & 1,08 & NS \\
\hline RCF (\%) & $53,27^{a}$ & $52,99^{a}$ & $53,20^{a}$ & $52,34^{a}$ & 1,03 & NS \\
\hline RBCC (\%) & $61,89^{a}$ & $60,81^{a}$ & $60,75^{a}$ & $61,58^{a}$ & 1,01 & NS \\
\hline RBCF (\%) & $59,80^{a}$ & $59,68^{a}$ & $59,51^{a}$ & $59,53^{a}$ & 0,92 & NS \\
\hline Cabeza (\%) & $4,82^{\mathrm{a}}$ & $4,99^{a}$ & $5,07^{a}$ & $4,81^{a}$ & 0,20 & NS \\
\hline Sangre (\%) & $2,84^{a}$ & $3,02^{a}$ & $3,27^{a}$ & $2,74^{\mathrm{a}}$ & 0,50 & NS \\
\hline Piel (\%) & $9,43^{a}$ & $9,81^{a}$ & $9,98^{a}$ & $8,89^{a}$ & 0,57 & NS \\
\hline Extremidades (\%) & $2,27^{b}$ & $2,34^{\mathrm{ab}}$ & $2,42^{a}$ & $2,29^{a b}$ & 0,07 & 0,0371 \\
\hline Vísceras verdes (\%) & $6,87^{a}$ & $6,78^{a}$ & $7,27^{a}$ & $7,69^{a}$ & 0,56 & NS \\
\hline Vísceras rojas (\%) & $5,40^{\mathrm{a}}$ & $5,08^{a}$ & $5,29^{a}$ & $5,33^{a}$ & 0,23 & NS \\
\hline Contenido Gl (\%) & $10,91^{a}$ & $11,20^{\mathrm{a}}$ & $10,62^{a}$ & $12,08^{a}$ & 0,93 & NS \\
\hline $\mathrm{pH}$ al sacrificio & $6,63^{a}$ & $6,42^{\mathrm{a}}$ & $6,40^{\mathrm{a}}$ & $6,42^{\mathrm{a}}$ & 0,17 & NS \\
\hline pH post mórtem & $5,59^{a}$ & $5,51^{a}$ & $5,73^{a}$ & $5,73^{a}$ & 0,18 & NS \\
\hline
\end{tabular}

$\mathrm{PVV}=$ peso vivo vacio; $\mathrm{PCC}=$ peso de la canal caliente; $\mathrm{PCF}=$ peso de la canal fría; $\mathrm{RCC}=$ rendimiento de la canal caliente; $R C F=$ rendimiento de la canal fría; $R B C C=$ rendimiento biológico de la canal caliente; $\mathrm{RBCF}=$ Rendimiento biológico de la canal fría; $\mathrm{DE}=$ desviación estándar; Sig.= significancia; ${ }^{* *} \mathrm{p}<0,01$; ${ }^{*} \mathrm{p}<0,05$.

${ }^{a b}$ En cada fila, cifras con letras distintas son significativamente diferentes. 


\section{RESENDIZ, HERNÁNDEZ, GUERRERO, GALLEGOS, MARTÍNEZ Y SÁNCHEZ}

2005), situación que explica los resultados obtenidos en el presente estudio. Además, la inclusión de alfalfa en la dieta, por su contenido y tipo de proteína condiciona mejores ganancias de peso (Marshall et al., 2001). No existen publicaciones relativas a la eficacia del uso de forrajes en el incremento del peso y rendimiento de canal o sobre el peso de los órganos (McClure et al., 2000), más allá de los estudios referentes a composición de la canal y sus propiedades organolépticas (Borton et al., 2005). Aunque el tamaño adulto del animal influye en el peso al mercado, y por tanto, puede estar involucrado en el rendimiento en canal (McDonald et al., 2006; Cabrera et al., 2007). Puede hipotetizarse, además, que los animales de este estudio presentaron mayor rendimiento en canal por el rápido crecimiento, donde el tiempo no fue el suficiente para incrementar la masa muscular en relación a la deposición de grasa, como sucede con los animales finalizados en pastoreo.

El pH de la canal al sacrificio y post mórtem fue de 6,47 y 5,64, respectivamente, valores ubicados dentro del rango normal, similares a los encontrados por Torrescano et al. (2009) y Hernández et al. (2009), quienes reportan en ovinos Pelibuey un $\mathrm{pH}$ al sacrificio de 6,10 y 6,34 , respectivamente, así como un valor de $\mathrm{pH}$ post mórtem de 5,80 y 5,53, respectivamente. Estos resultados indican que los animales no sufrieron algún tipo de estrés durante el proceso desde el transporte hasta el sacrificio, factor importante a considerar, ya que la relación entre el pH y el estrés están fuertemente involucradas en la calidad de la carne (Hargreaves et al., 2004).

\section{ANÁLISISECONÓMICO}

En la tabla $\mathbf{V}$ se presenta el análisis económico de la alimentación. Es importante señalar que el uso de la alfalfa en la alimentación de los borregos surge de la necesidad de disminuir los costos de alimentación al reducir la utilización de granos por su incremento constante en el precio. El análisis económico muestra que la dieta que no contenía alfalfa presentó el mayor costo

Tabla $\boldsymbol{V}$. Análisis económico por animal por concepto de alimentación (US dólares), cuando se incluyen diferentes niveles de alfalfa en la dieta para corderos Pelibuey. (Economic analysis per animal based on feeding concept (US dollars), when including different levels of alfalfa in the Pelibuey lambs diet).

\begin{tabular}{lcccc}
\hline & \multicolumn{4}{c}{ Tratamientos (\% de alfalfa) } \\
& 0 & 20 & 30 & 40 \\
\hline Costos por alimentación & & & & \\
Duración de la engorda (días) & 74 & 74 & 74 & 74 \\
Costo de la dieta (\$/kg) & 0,29 & 0,27 & 0,26 & 0,25 \\
CMS (kg/día/animal) & 1,20 & 1,19 & 1,36 & 1,39 \\
Costo del kg CMS/día/animal (\$) & 0,34 & 0,32 & 0,35 & 0,34 \\
Costo total (\$) & 25,75 & 23,75 & 26,19 & 25,67 \\
Retornos & & & & \\
PGT (kg/animal) & 15,39 & 15,18 & 15,66 & 16,50 \\
Precio del kg en pie (\$) & 2,45 & 2,45 & 2,45 & 2,45 \\
Ingreso por venta de carne/animal (\$) & 37,70 & 37,19 & 38,37 & 40,42 \\
Ingreso neto & 11,95 & 13,44 & 12,18 & 14,75 \\
& & &
\end{tabular}

$\$ / \mathrm{kg}=$ costo por kilogramo de alimento; $\mathrm{CMS}=$ consumo de materia seca; $\mathrm{PGT}=$ peso ganado total $\$ \$=$ costo por kg de alimento. Precio del dólar=12,24 pesos mexicanos. 


\section{USO DE LA ALFALFA EN LA ALIMENTACIÓN DE CORDEROS PELIBUEY}

por kilogramo de alimento $(\$ 0,29)$, seguido de la dieta con $20 \%(\$ 0,27), 30 \%(\$ 0,26)$ y $40 \%(\$ 0,25)$ de alfalfa, respectivamente. Estas diferencias son explicadas por la disminución de la cantidad de sorgo y pasta de soya. Con el incremento de 20 a $40 \%$ de alfalfa, los ingresos netos por concepto de la venta, incrementan de $\$ 13,44$ a $\$ 14,75$ respectivamente, en comparación con la dieta que no la contenía, que fue de $\$ 11,95$, representando un incremento de 11 y $19 \%$, respectivamente. Los resultados están referidos con base a un animal, durante el periodo de engorda. Este escenario sugiere que la alfalfa puede ser una opción para mejorar la rentabilidad en la engorda de corderos, incluyendo hasta $40 \%$ y reduciendo el nivel de sorgo y pasta de soya en la dieta.

\section{CONCLUSIONES}

Los resultados en las variables evalua-

\section{BIBLIOGRAFIA}

Álvarez, J.H.; Dichio, L.; Pece, A.M.; Cangiano, A.C. y Galli, R.J. 2006. Producción de leche bovina con distintos niveles de asignación de pastura y suplemento energético. Cien Inv Agr, 33: 99-107.

Álvarez, M.G.; Melgarejo, V.L. y Castañeda, N.Y. 2003. Ganancia de peso, conversión y eficiencia alimentaria en ovinos alimentados con fruto (semilla con vaina) de parota (Enterolobiumcyclocarpum) y pollinaza. Rev Vet Méx, 34: 39-46.

AOAC. 1984. Official Methods of Analysis. 5a ed. Association of Official Analytical Chemists. Washington, DC. EE.UU.

Avendaño, R.J.; Fernández, E.F.; Ovalle, M.C. y Blu, L.F. 2004. Ovinos alimentados con raciones que incluyen tagasaste (Chamaecytisus proliferus subsp. Palmensis) en reemplazo de heno de alfalfa. II. Digestibilidad y consumo de nutrientes. Agric Téc, 64: 271-279.

Barahona, R.R. y Sánchez, P.S. 2005. Limitaciones físicas y químicas de la digestibilidad de pastos tropicales y estrategias para aumentarla. Corpoica, 6: 69-82.

Bhatti, S.A.; Bowman, J.G.P.; Firkins, J.L.; Grove, das fueron similares, esto sugiere que la alfalfa como ingrediente en la dieta de corderos en finalización puede mejorar la rentabilidad, sin afectar las variables productivas y de la canal. La alfalfa puede ser considerada como un ingrediente base en la alimentación ovina, incluyendo hasta $40 \%$ de la materia seca en la dieta. Sin embargo, más investigación es necesaria, para confirmar estos resultados.

\section{AGRADECIMIENTOS}

Al Consejo Nacional de Ciencia y Tecnología (CONACyT)-México por la beca otorgada para los estudios de Maestría del primer autor, y a la Universidad Autónoma Chapingo-México (Módulo de Producción Ovina), por el apoyo para la realización de esta investigación, así como al Fideicomiso 167304 del Colegio de Postgraduados por financiar parcialmente esta investigación.

A.V. and Hunt, C.W. 2008. Effect of intake level and alfalfa substitution for grass hay on ruminal kinetics of fiber digestion and particle passage in beef cattle. J Anim Sci, 86: 134-145.

Borton, R.J.; Loerch, S.C.; McClure, K.E. and Wulf, D.M. 2005. Comparison of characteristics of lambs fed concentrate or grazed on ryegrass to traditional or heavy slaughter weights. I. Production, carcass, and organoleptic characteristics. J Anim Sci, 83: 679-685.

Boschini, C. 2001. Degradabilidad in situ de la materia seca, proteína y fibra del forraje de morera (Morus alba). Agron Mesoam, 12: 7987.

Brito, A.F.; Tremblay, G.F.; Lapierre, H.; Bertrand, A.; Castonguay, Y.; Bélanger, G.; Michaud, R.; Benchaar, C.; Ouellet, D.R. and Berthiaume, R. 2009. Alfalfa cut at sundown and harvested as baleage increases bacterial protein synthesis in late-lactation dairy cows. J Dairy Sci, 92: 1092-1107.

Church, D.C.; Pond, K.R. y Pond, W.G. 2007. Fundamentos de nutrición y alimentación de animales. Editorial Limusa S.A. de C.V. Méxi- 


\section{RESENDIZ, HERNÁNDEZ, GUERRERO, GALLEGOS, MARTÍNEZY SÁNCHEZ}

co. pp. 344-345.

Cabrera, N.A.; Rojas, M.P.; Renteria, D.I.; Serrano, S.A. y López, O.M. 2007. Influencia de la suplementación sobre la ganancia de peso y calidad de la canal en borregos Dorper/Katahdin. Revista UDO Ag, 7: 245-251.

Distel, R.A. y Villalba, J.J. 2007. Diversidad vegetal, selección de dieta y producción animal. Rev Argentina Prod Anim, 27: 55-63.

Fernández, E.F.; Avendaño, R.J.; Ovalle, M.C.; Fraga, S.A. y Blu, L.F. 2004. Ovinos alimentados con raciones que incluyen tagasaste (Chamaecytisus proliferus subsp. palmensis) en reemplazo de heno de alfalfa. I. Consumo y variaciones de peso. Agric Téc, 64: 264-270.

Getachew, G.P.; Depeters, E.J.; Pittroff, W.; Putnam, D.H. and Dandekar, A.M. 2006. Review: Does protein in alfalfa need protection from rumen microbes? The Professional Animal Scientist, 22: 364-373.

García, de M.E. 1989. Modificaciones al sistema de clasificación climática de Köppen. 3. ${ }^{\text {a } e d . ~ U N A M . ~}$ Instituto de Geografía. México, D.F. 276 pp.

Hargreaves, A.; Barrales, L.; Peña, I.; Larraín, R. y Zamorano, L. 2004. Factores que influyen en el $\mathrm{pH}$ último e incidencia de corte oscuro en canales de bovinos. Cien Inv Agr, 31: 155-166.

Haugen, H.L.; Ivan, S.K.; MacDonald, J.C. and Klopfenstein, T.J. 2006. Determination of undegradable intake protein digestibility of forages using the mobile nylon bag technique. J Anim Sci, 84: 886-893.

Hernández, C.L.; Ramírez, B.J.E.; Guerrero, L.M.I.; Hernández, M.O.; Crosby, G.M.M. and Hernández, C.L.M. 2009. Effects of crossbreding on carcass and meat quality of Mexican lambs. Arq Bras Med Vet Zoo, 61: 475-483.

Jahn, B.E.; Vidal, V.A. y Soto, O.P. 2000. Sistema de producción de leche basado en alfalfa (Medicago sativa) y maíz (Zea mays) para la zona centro sur. II Consumo y calidad del forraje. Agric Téc, 60: 99-111.

Jaramillo, L.E.; Molinar, H.F.; Leos, M.J.A. eHinojosa, A.M.C. 2008. Efecto de la dieta en corderos de lana y pelo sobre la ganancia diaria de peso, consumo de alimento, conversión alimenticia y características de la canal. Ciencia en la frontera: revista de ciencia y tecnología de la UACJ, 6: $131-139$

Krizsan, S.J. and Randby, A.T. 2007. The effect of fermentation quality on the voluntary intake of grass silage by growing cattle fed silage as sole feed. J Anim Sci, 85: 984-996.

Larraín, R.E.; Schaefer, D.M.; Arp, S.C.; Claus, J.R. and Reed, J.D. 2009. Finishing steers with diets based on corn, high-tannin sorghum, or a mix of both: Feedlot performance, carcass characteristics and beef sensory attributes. J Anim Sci, 87: 2089-2095.

Loncke, C.; Ortigues, M.I.; Vernet, J.; Lapierre, H.; Sauvant, D. and Noziere, P. 2009. Empirical prediction of net portal appearance of volatile fatty acids, glucose, and their secondary metabolites ( $\beta$-hydroxybutyrate, lactate) from dietary characteristics in ruminants: A metaanalysis approach. J Anim Sci, 87: 253-268.

López, P.M.G.; Rubio, L.M.S. y Valdés, M.S.E. 2000. Efecto del cruzamiento, sexo y dieta en la composición química de la carne de ovinos Pelibuey con Rambouillet y Suffolk. Rev Vet Méx, 31: 11-19.

Marshall, S.W.; Bertot, V.J.A.; Delgado, A.; Collantes, C.M. y Alba, C. 2001. Características productivas y rasgos de la canal de corderos Pelibuey alimentados con heno y suplementados con gallinaza y harina de soya. Rev Prod Anim, 13: 43-47.

Martin, J.M. and Rogers, R.W. 2004. Review: Forage-produced beef: Challenges and potential. The Professional Animal Scientist, 20: 205-210.

Martínez, V.I.; Periago, M.J. y Ros, G. 2000. Significado nutricional de los compuestos fenólicos de la dieta. Arch Latinoam Nutr, 50: 5-18.

Medrano, J.A. 2000. Recursos animales locales del centro de México. Arch Zootec, 49: 385-390.

Mendiola, G.A.; Martínez, H.P.A.; Cortés, D.E. y Sánchez, D.C. 2007. Efecto del pastoreo mixto y monoespecífico de una pradera de alfalfaovillo. Agrociencia, 41: 395-403.

McClure, K.E.; Solomon, M.B. and Loerch, S.C. 2000. Body weight and tissue gain in lambs fed an all-concentrate diet and implanted with trenbolone acetate or grazed on alfalfa. J Anim Sci, 78: 1117-1124.

McDonald, P.; Edwards, R.A.; Greenhalgh, J.F.D. y Morgan, C.A. 2006. Nutrición animal. Editorial Acribia S.A. 6. ${ }^{a}$ edición. Zaragoza, España. 581 pp.

Moss, B.R.; Lin, J.C.; Pas, D.; Wright, D.; Wright, L.;

Archivos de zootecnia vol. 62, núm. 239, p. 466. 


\section{USO DE LA ALFALFA EN LA ALIMENTACIÓN DE CORDEROS PELIBUEY}

Mcelhenney, W.H.; Prevatt, J.W. and Dorough, H.D. 2002. Milk production from cows fed corn, alfalfa, or ryegrass silage ensiled in conventional, vacuum or packed systems. The Profesional Animal Scientist, 18: 324-331.

Mora, J.G., Bárcena, G.R., Mendoza, M.G., González, M.S. y Herrera, H.J. 2002. Respuesta productiva y fermentación ruminal en borregos alimentados con grano de sorgo tratado con amilasas. Agrociencia, 36: 31-39.

Morales, M.M.; Martínez, D.J.P.; Torres, H.G. y Pacheco, V.J.E. 2004. Evaluación del potencial para la producción ovina con el enfoque de agroecosistemas en un ejido de Veracruz, México. Téc Pecu Méx, 42: 347-359.

Murray, K.R.; Granner, K.D. y Rodwell, W.V. 2007. Harper. Bioquímica ilustrada. Editorial: El Manual Moderno, S.A. de C.V. $17^{a}$ edición. México, D.F.

NRC. 2007. Nutrient requeriments of sheep. National Academy Press. $6^{\text {th }}$ Rev Ed. Washington, D.C. USA.

Ojeda, A.; Frías, A.; González, R.; Linares, Z. y Pizzani, P. 2010. Contenido de taninos, fósforo fítico y actividad de fitasas en el grano de 12 híbridos de sorgo granífero (Sorghum bicolor (L) Moench). Arch Latinoam Nutr, 60: 93-98.

Otero, J.M. e Hidalgo, L.G. 2004. Taninos condensados en especies forrajeras de clima templado: efecto sobre la productividad de rumiantes afectados por parasitosis gastrointestinales (una revisión). Livest Res Rural Dev, 16 (2). http://www.Irrd.org//rrd16/2/oter1602.htm (04/ 11/2009).

Partida, P.J.; Braña, V.D. y Martínez, R.L. 2009. Desempeño productivo y propiedades de la canal en ovinos Pelibuey y sus cruzas con Suffolk o Dorset. Téc Pecu Méx, 47: 313-322.

Pérez, A.; Saucedo, O.; Iglesias, J.; Wencomo, H.B.; Reyes, F.; Oquendo, G. y Milián, I. 2010. Caracterización y potencialidades del grano de sorgo (Sorghum bicolor L. Moench). Pastos y Forrajes, 33: 1-17.

Phillips, W.A.; Rao, S.C.; Fitch, J.Q. and Mayeux,
H.S. 2002. Digestilility and dry matter intake of diets containing alfalfa and kenaf. J Anim Sci, 80: 2989-2995.

Ramírez, R.O.; Pérez, P.J.; Hernández, G.A.; Herrera, H.J.G. y Martínez, H.P.A. 2003. Evaluación del rendimiento y la utilización de la asociación estrella-clitoria cosechada a diferente asignación de forraje. Téc Pecu Méx, 41: 219230.

SAGARPA. 2010. Producción anual pecuaria en México. Centro de Estadística Agropecuaria. http://www.sagarpa.gob.mx/ganaderia/publi cacion.htm (10/03/2009).

SAS. 1999. User's guide: Statiscs. SAS Institue. Cry, North Carolina. USA.

SIAP. 2010. Anuario estadístico de la producción agrícola en México. http://www.siap.gob.mx/ (19/12/2009).

Steel, G.D.R. y Torrie, H.J. 1989. Bioestadística: principios y procedimientos. $1 .^{\mathrm{a}} \mathrm{ed}$. en español. McGraw-hill/Interamericana S.A de C.V. México. 622 pp.

Tablada, A.S.R.; Martínez, H.P.A.; Sánchez, R.C. y Cortes, D.E. 2003. Rebrote en alfalfa-ovillo bajo pastoreo mixto durante el invierno. FCVLUZ, 13: 312-318.

Torrescano, U.G.; Sánchez, E.A.; Peñuri, M.F.; Velázquez, C.J. y Sierra, R.T. 2009. Característica de la canal y calidad de la carne de ovinos Pelibuey, engordados en Hermosillo, Sonora. Biotecnia, 11: 41-50.

Van Soest, P.J.; Robertson, J.B. and Lewis, B.A. 1991. Methods for dietary fiber, neutral detergent fiber, and nonstarch polysaccharides in relation to animal nutrition. Symposium: Carbohydrate Methodology, Metabolism, and Nutritional Implications in Dairy Cattle. J Dairy Sci, 74: 3583-3597.

Whetsell, M.S.; Prigge, E.C. and Nestor, E.L. 2004. Influence of mass of ruminal contents on voluntary intake and digesta passage in steers fed a forage and a concentrate diet. J Anim Sci, 82: 1806-1817. 